\title{
Directing Brownian motion by oscillating barriers
}

\author{
S. Bleil, ${ }^{1}$ P. Reimann, ${ }^{2}$ and C. Bechinger ${ }^{1}$ \\ 12. Physikalisches Institut, Universität Stuttgart, 70550 Stuttgart, Germany \\ ${ }^{2}$ Fakultät für Physik, Universität Bielefeld, 33615 Bielefeld, Germany
}

(Received 22 November 2006; published 26 March 2007)

\begin{abstract}
We consider the Brownian motion of a colloidal particle in a symmetric, periodic potential, whose potential barriers are subjected to temporal oscillations. Experimentally, the potential is generated by two arrays of trapped, negatively charged particles whose positions are periodically modulated with light forces. This results in a structured channel geometry of locally variable width. If all potential barriers are oscillating in synchrony, a resonancelike peak of the effective diffusion coefficient upon variation of the oscillation period is observed. For asynchronously oscillating barriers, the particle can be steered with great reliability into one or the other direction by properly choosing the oscillation periods of the different barriers along the channel.
\end{abstract}

DOI: 10.1103/PhysRevE.75.031117

PACS number(s): 05.40.-a, 05.60.Cd, 82.70.Dd, 05.60.-k

\section{INTRODUCTION}

Brownian motion in quasi-one-dimensional channels is a recurrent theme in a variety of different contexts, such as molecular transport through cell membranes [1], single-file diffusion [2], and transport by various kinds of noise-assisted rectification, pumping, or ratchet effects [3]. Here, we introduce a transport mechanism for a Brownian particle in a temporally modulated channel that is closely related to the above examples but still fundamentally different from all previous studies as far as the basic physical principles are concerned. Specifically, we demonstrate how a particle can be directed inside a channel with high fidelity toward one or the other end by subjecting the cross section of the channel to time-dependent, but spatially symmetric modulations.

The plan of the paper is as follows: We start with the description and characterization of the experimental system. Next, we focus on the case of synchronously oscillating potential barriers within the channel with particular emphasis on the phenomenon of resonant activation and the effective diffusion coefficient. In the case of asynchronously oscillating barriers, we demonstrate systematic particle transport whose direction can be controlled by a suitable choice of the oscillation periods. Finally, we point out the main differences and similarities with the above-mentioned previously established transport mechanisms.

\section{EXPERIMENTAL SYSTEM}

As a sample cell we used a thin glass cuvette which contained an aqueous suspension of negatively charged silica beads with $2 r \simeq 1.57 \mu \mathrm{m}$ diameter, interacting via a screened Yukawa potential [4]. Due to gravity and the negatively charged silica plates forming the sample cell the particle motion is confined to a horizontal plane where they undergo Brownian motion [5]. In contrast to earlier experiments, where static one-dimensional channels were achieved by lithographic methods [6] or continuously scanned optical tweezers [7], here we are interested in the effect of fluctuating channels on the behavior of a probe particle located inside the channel. Therefore, we followed another approach to create channels whose geometry can be dynamically modu- lated: with a scanning optical tweezer based on an acoustooptical deflection system (for details see [8]) we create two parallel arrays (distance $b \simeq 8.5 \mu \mathrm{m}$ ) of equally spaced ( $a$ $\simeq 3.9 \mu \mathrm{m}$ ) laser spots which serve as narrow, steep optical traps for colloidal particles [Fig. 1(a)]. Once the traps are filled with particles (channel particles), they form a channellike structure whose spatial and temporal behavior can be conveniently controlled. The trapping strength of the channel particles is about $30 k_{B} T$ which is considerably larger than the electrostatic interaction of adjacent particles. Therefore the channel geometry is entirely determined by the position of the laser tweezers.

In order to study the effect of channel fluctuations on the motional behavior of a probe particle located inside the channel, we subject the channel particles to a periodic modulation in the $y$ direction with period $\tau$ and amplitude $0.5 \mu \mathrm{m}$ (for simplicity, we apply those oscillations only to one channel wall while the other wall remains at rest). Depending on whether all channel particles are modulated in phase and with the same period $\tau$, the resulting time-dependent potential for the probe particle upon the channel modulation leads to symmetric or asymmetric spatial potentials [Fig. 1(b)]. Due to the strong electrostatic interaction, the motion of the probe particle is to good approximation one dimensional. Therefore, in the following only the motional behavior along the $x$ direction will be considered.

In the following we investigate how the motional behavior of a single probe particle is affected by different time-

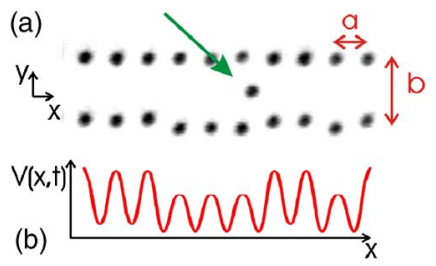

FIG. 1. (Color online) (a) Snapshot of colloidal particles which form a one-dimensional channel for another free particle (arrow). The channel particles are localized with a scanning optical tweezer which allows one to modulate the geometry of the channel. (b) Sketch of the instantaneous effective potential $V(x, t)$ acting on the probe particle along the $x$ axis due to its interaction with the channel particles. 
dependent fluctuations of the channel geometry. For a single experimental run, the probe particle is first positioned with an optical tweezer in the center of the channel and then released. The particles are imaged with an inverted microscope onto a charge coupled device (CCD) camera, and the trajectories are monitored by means of digital video microscopy. To avoid boundary effects, in the following we only consider the behavior of the probe particle in the central channel region where boundary effects can be neglected. Once the probe particle leaves the channel, it is reset back to the channel center. Because the channel modulation phase was not reset after each initialization, this procedure also leads to a phase averaging.

\section{SYNCHRONOUSLY OSCILLATING BARRIERS}

\section{A. Resonant activation}

We start with the case where the channel particles are subjected to a synchronous motion with the same period $\tau$, resulting in an effective potential $V(x, t)$ which is at any given time instant $t$ spatially periodic and symmetric (apart from boundary effects at the channel ends) and whose potential barriers switch between a "high" and a "low" value (compared to $k_{B} T$ ) after every half-period $\tau / 2$. A typical trajectory $x(t)$ of the probe particle is given in Fig. 2(a).

In order to evaluate the averaged jumping time of a particle between neighboring potential wells, we first applied suitable thresholds to map the particles trajectory to a system with discrete states [green dashed line in Fig. 2(a)]. The vertical lines describe the modulation of the channel. Solid (dotted) lines indicate the time where the channel switches to a period with high (low) potential $V(x, t)$. Most of the jumps between individual minima of $V(x, t)$ occur when the channel switches from the low to the high barrier phase. From this, we determined the mean first passage time (MFPT) $\mathcal{T}$ as a function of the oscillation period $\tau$ [Fig. 2(b)]. The pronounced minimum of $\mathcal{T}$ at about $\tau=35 \mathrm{~s}$ is closely related (though not identical in detail) to the resonant activation effect [9] and explained as follows:

During the first half-period $\tau / 2$ the barriers are "low" (compared to $k_{B} T$ ) and the particle roughly exhibits free thermal diffusion with the experimentally determined diffusion coefficient $D \simeq 0.24 \mu \mathrm{m}^{2} / \mathrm{s}$ [5]. Hence, starting from a potential minimum, the necessary time to travel the distance $a / 2$ to one of the adjacent maxima is about $(a / 2)^{2} / 2 D$. If at this moment the barrier turns to its "high" value, the particle can cover the remaining distance $a / 2$ to the next minimum by drifting down along the potential slope. Assuming that the drift is much faster than the diffusion time, the cooperation of diffusion and deterministic drift leads to a maximal reduction of the MFPT $\mathcal{T}$ if the duration of the low barrier phase $\tau / 2$ matches the diffusion time $(a / 2)^{2} / 2 D$. In other words, the minimum of $\mathcal{T}$ is predicted to roughly occur at

$$
\tau_{\text {min }} \approx a^{2} / 4 D \text {. }
$$

For our experimental parameter values $a \simeq 3.9 \mu \mathrm{m}$ and $D$ $\simeq 0.24 \mu \mathrm{m}^{2} / \mathrm{s}$ this yields $\tau_{\min } \approx 15.8 \mathrm{~s}$. In face of the above very rough estimate and the fact that even in the "low" state
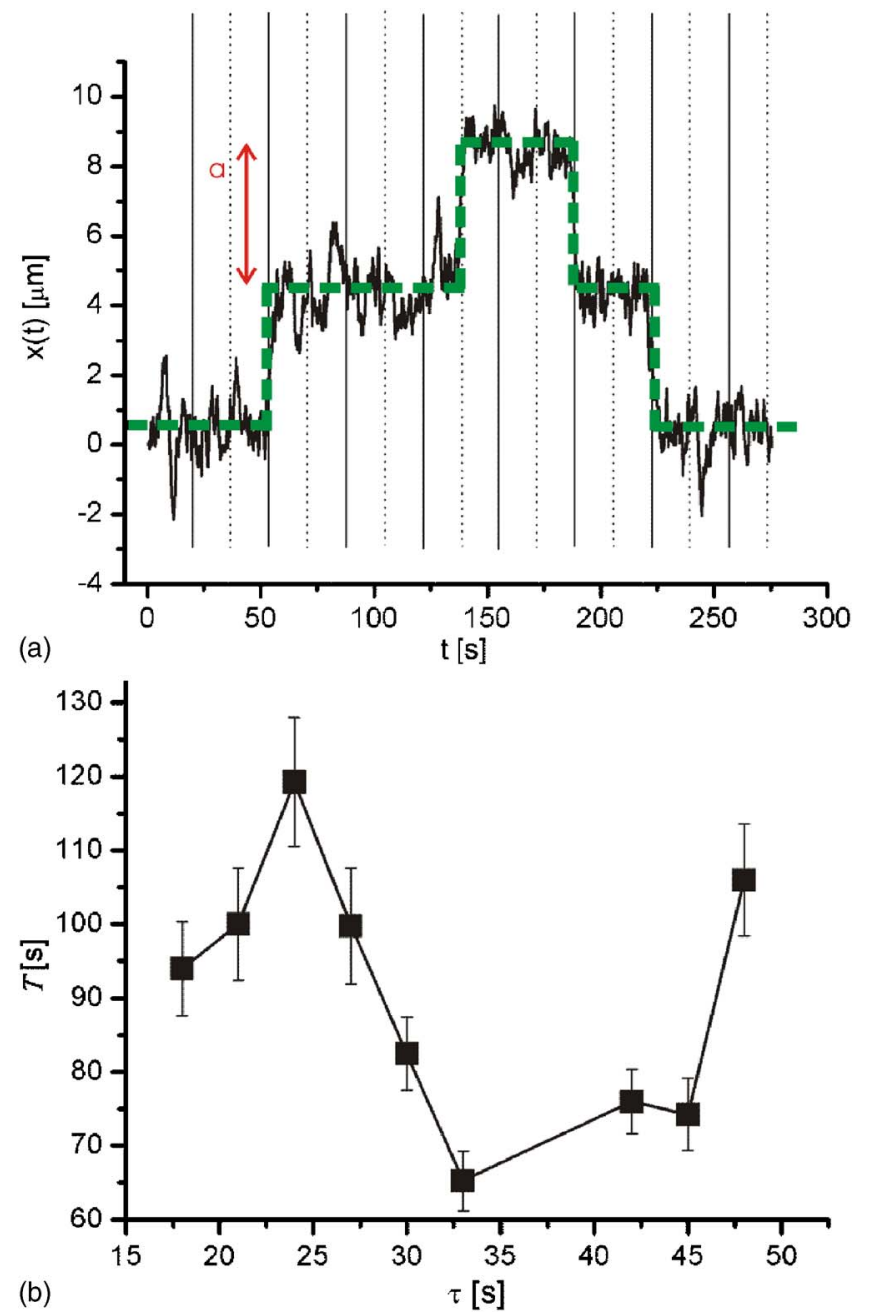

FIG. 2. (Color online) (a) Typical raw trajectory $x(t)$ of the probe particle for synchronously oscillating channel particles in Fig. 1(a). The trajectory is mapped to a discrete-state system (green dashed line) by applying suitable thresholds. From the time intervals represented by the horizontal parts of the green dashed line we obtain the first passage times from one potential minimum to the next. By averaging, one obtains the mean first passage time (MFPT) $\mathcal{T}$. To avoid deviations from spatial periodicity, transitions between minima very close to the channel ends are not included in the average. The vertical lines describe the modulation of the channel. Solid (dotted) lines indicate the time where the channel switches to high (low) potential $V(x, t)$. (b) Experimentally determined MFPT $\mathcal{T}$ for various oscillation periods $\tau$.

the particle does not diffuse entirely freely, this is in reasonable agreement with the experimentally observed value $\tau_{\min }$ $\approx 35 \mathrm{~s}$ in Fig. 2(b). Since the particle reaches the next minimum at the end of the "high" barrier phase only with a probability of roughly $1 / 2$ (with the same probability it returns to the same minimum from where it started out), we obtain

$$
\mathcal{T}\left(\tau_{\min }\right) \approx 2 \tau_{\text {min }},
$$

which is in good agreement with Fig. 2(b). For very fast oscillations, the particle experiences the time-averaged potential $V_{0}(x)$ [10]. Hence, a lower estimate for $\mathcal{T}$ is the free 
diffusion time $a^{2} / 2 D$ over one spatial period $a$. The same lower estimate obviously also applies for very slow oscillations. In other words, both for large and small $\tau$ the MFPT $\mathcal{T}$ is at least twice as large as for $\tau=\tau_{\min }$, again in agreement with Fig. 2(b). Without going into the details, one can furthermore see that this mechanism works best in the sense that the minimum of $\mathcal{T}$ will be most pronounced if the transitions across the high barriers are negligibly rare, while the low barriers are of negligible height compared to $k_{B} T$. Both requirements are roughly but not perfectly met by the actual experiment in Figs. 1 and 2.

In addition to the minimum, in Fig. 2(b), we also observe a maximum of $\mathcal{T}$ at $\tau \approx 24 \mathrm{~s}$. Though a maximum may appear reminiscent of a special type of resonant activation unraveled in [11], this point needs further investigation which is beyond the scope of this paper.

\section{B. Resonant enhancement of diffusion}

In this section we are interested in the effective diffusion coefficient $D_{\text {eff }}$ of the probe particle in a synchronously oscillating potential $V(x, t)$. It is directly obtained from the particle trajectory $x(t)$ according to

$$
D_{e f f}:=\lim _{t \rightarrow \infty} \frac{\left\langle x^{2}(t)\right\rangle-\langle x(t)\rangle^{2}}{2 t} .
$$

Without oscillations-i.e., for a static channel of width $8.5 \mu \mathrm{m}$-we experimentally found $D_{\text {eff }}^{\text {static }} \simeq 0.16 \mu \mathrm{m}^{2} / \mathrm{s}$, which is, as expected [12], considerably smaller than the free diffusion coefficient $D \simeq 0.24 \mu \mathrm{m}^{2} / \mathrm{s}$ [5]. The almost instantaneous transitions between neighboring potential minima in Fig. 2(a) indicate that the particle dynamics can be described in good approximation as a sequence of independent hopping events between discrete states. The corresponding hopping rate $\gamma$ between neighboring states then readily follows as the inverse of the MFPT $\mathcal{T}$. Moreover, for the effective diffusion coefficient (3) in an $a$-periodic hopping dynamics one recovers the common relation $D_{e f f}=\gamma a^{2}$. In other words, we obtain the following approximative expression for the effective diffusion coefficient:

$$
D_{\text {eff }}=a^{2} / \mathcal{T}
$$

see Fig. 3. For one oscillation period-namely, $\tau=30 \mathrm{~s}$, we also have carried out a quite time-consuming direct experimental measurement of the effective diffusion coefficient (3), yielding $D_{\text {eff }}=0.19 \mu \mathrm{m}^{2} / \mathrm{s}$. Comparison with Fig. 3 implies that our above indirect results for $D_{\text {eff }}$ are in excellent agreement and thus the analytical approximation is indeed applicable.

According to Eq. (4) or by comparison of Figs. 2(b) and 3 we see that the "resonant activationlike" minimum of the MFPT translates into an equivalent "resonancelike" peak of the effective diffusion coefficient in an array of oscillating barriers, which can be controlled by the period $\tau$ of those oscillations. The basic physical mechanism for this selective diffusion enhancement is once again the above-discussed cooperation of free thermal diffusion and deterministic relaxation, somewhat similar to the theoretical ideas in [13].

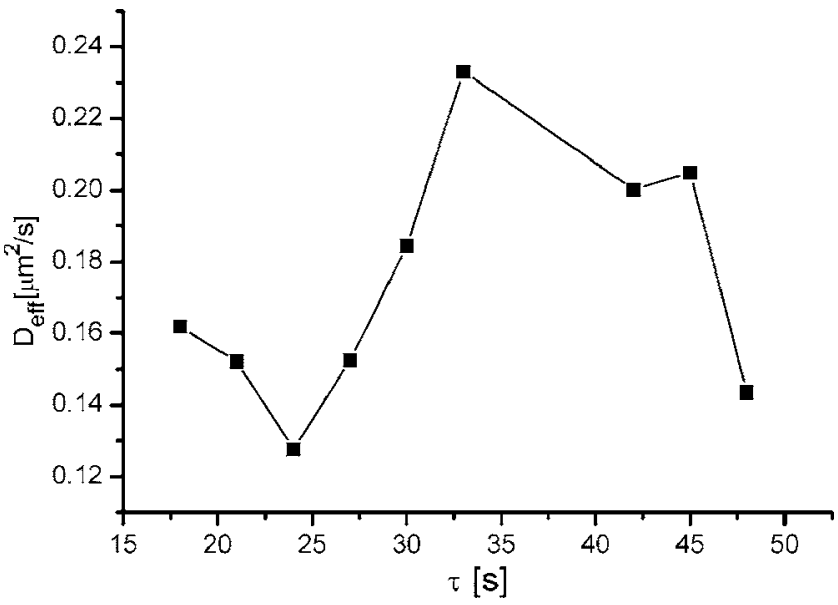

FIG. 3. Theoretical approximation (4) for the effective diffusion coefficient $D_{\text {eff }}$ vs oscillation period $\tau$ for the same system as in Fig. 2.

The maximal value of $D_{\text {eff }}$ is almost identical with the free diffusion coefficient $D \simeq 0.24 \mu \mathrm{m}^{2} / \mathrm{s}$ [5], but there can be little doubt that for other experimental parameters than in Fig. 3 one could also reach effective diffusion coefficients considerably larger than $D$. Furthermore, instead of varying the oscillation period $\tau$, one could fix $\tau$ to its maximum value in Fig. 3 and instead vary some other quantity - for instance, the particle radius (and hence $D[5]$ ). Upon variation of this quantity, one will again find a "resonancelike" maximum of the effective diffusion coefficient. The corresponding difference in the diffusion coefficient for different particle species could be readily exploited to separate them from each other: Starting with a homogeneous mixture of particles, those with large effective diffusion coefficients would quickly "evaporate" out of the much more inert bulk of particles with low $D_{\text {eff }}$.

For very small modulation times $\tau$ the effective diffusion coefficient in Fig. 3 approaches quite closely the abovementioned finding of $D_{\text {eff }}^{\text {static }} \simeq 0.16 \mu \mathrm{m}^{2} / \mathrm{s}$ for a static periodic potential $V_{0}(x)$ corresponding to the average width $b$ $=8.5 \mu \mathrm{m}$ of the channel in Fig. 1(a). This indicates that the particles indeed effectively experience the average potential $V_{0}(x)$ for sufficiently fast oscillations, in spite of the fact that the potential is a nonlinear function of the channel width $b$, and hence the average potential in general need not agree very well with the static potential corresponding to the average width $b=8.5 \mu \mathrm{m}$.

\section{DIRECTED TRANSPORT FOR INCOHERENTLY OSCILLATING BARRIERS}

In this section we consider the case where all oscillating channel particles are jumping back and forth with different periods $\tau$, but still identical amplitudes $(0.5 \mu \mathrm{m})$. Specifically, we focus on the example that the period $\tau$ is increasing in constant steps $\Delta \tau$ when proceeding from one channel particle to its right neighbor in Fig. 1(a). Hence, the resulting potential $V(x, t)$ acting on the probe particle in Fig. 1(a) exhibits faster and faster potential barrier oscillations as the spatial argument $x$ increases. 

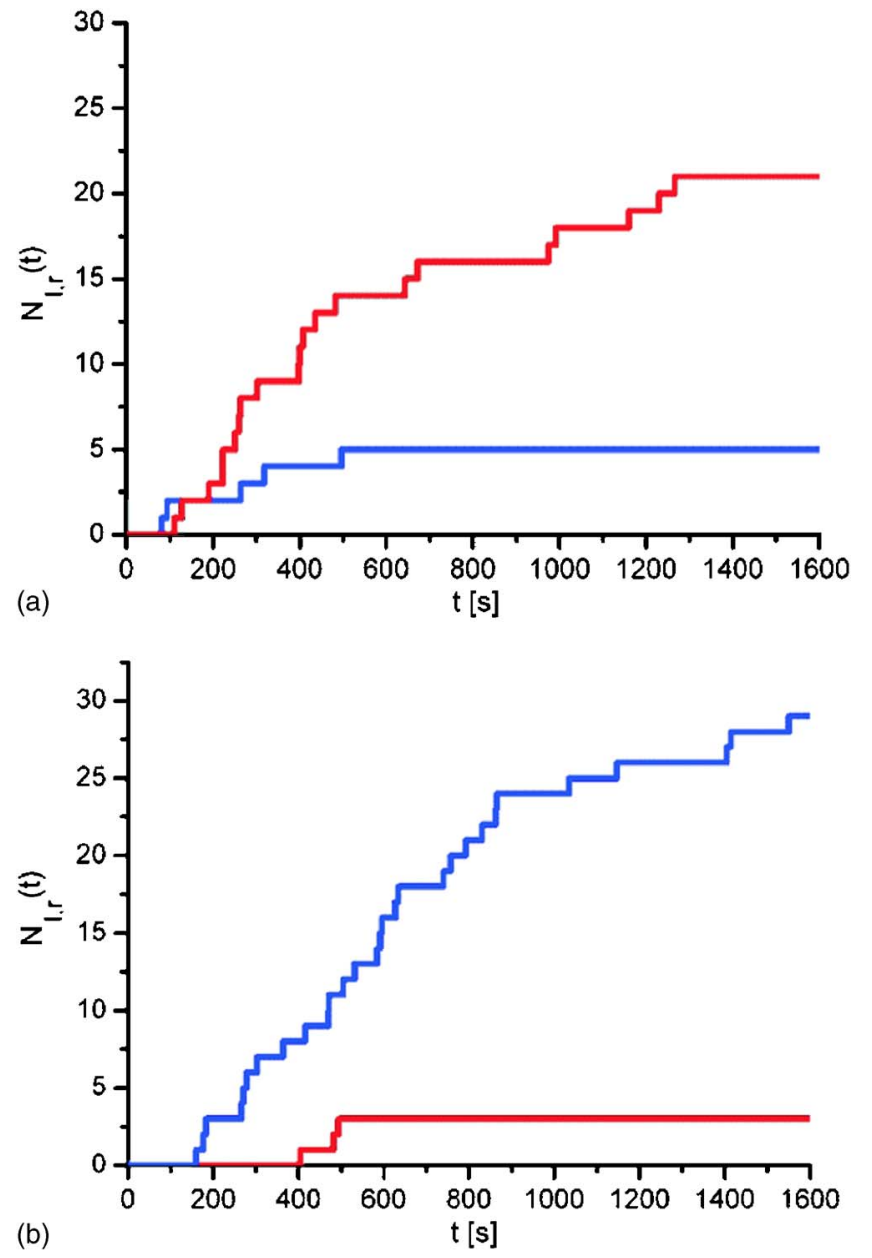

FIG. 4. (Color online) Total number of realizations $N_{l}(t)$ [red, upper curve in (a), lower curve in (b)] and $N_{r}(t)$ [blue, lower curve in (a), upper curve in (b)] which have left the channel until time $t$ through the left and right ends, respectively, starting at time $t=0$ in the middle of the channel. (a) Channel consisting of 11 oscillating channel particles with periods $\tau=30,33,36, \ldots, 60 \mathrm{~s}$ [from left to right in Fig. 1(a)]. (b) Twelve oscillating channel particles with periods $\tau=16,18,20, \ldots, 38 \mathrm{~s}$.

As detailed in Sec. II, the probe particle is repeatedly initialized in the middle of the channel and monitored until it leaves the channel either at the left or the right end. Figure 4 depicts our experimental results for the quantities $N_{l}(t)$ and $N_{r}(t)$, defined as the number of realizations which have left the channel until time $t$ through the left and right ends, respectively. Hence, at every time instant $t$ at which a realization exits to the left [right], $N_{l}(t)\left[N_{r}(t)\right]$ are increased by 1 . Accordingly, the final values $N_{l, r}\left(t_{f}\right)$ are the total number of exits to the left and right, respectively. Hence, $N_{l}\left(t_{f}\right)+N_{r}\left(t_{f}\right)$ are the total number of experimental realizations and $N_{l}\left(t_{f}\right) / N_{r}\left(t_{f}\right)$ is the relative probability for exiting left compared to right-i.e., a suitable quantifier of how reliably our channel device directs the particles in one or the other direction. In Fig. 4 the depicted time range slightly exceeds the value $t_{f}$, beyond which $N_{l, r}(t)$ will not change any more.

From Fig. 4 it is immediately obvious that the Brownian motion of a colloidal particle can be directed with high fi- delity either to the right or left end of the channel by suitably chosen oscillation periods $\tau$. To understand this behavior, it is important to recall that the MFPT depends on the oscillation period and thus changes along the channel. In the case of Fig. 4(a), the mean first passage times over the oscillating potential barriers increases from left to right according to Fig. 2(b). Within the hopping model developed in Sec. III B, this means that for every given discrete state (representing a potential minimum), transitions to the adjacent state to the left are more likely than to the right. Hence, a particle starting in the middle of the channel will preferably move to the left. Similarly, in the case of Fig. 4(b), the mean first passage times increases from the right to the left and thus results in a preferential motion to the right.

According to the above-mentioned discrete-state model, the situation is essentially equivalent to the motion in a static potential landscape with equally spaced minima but with static potential barriers between the minima which are either increasing (from left to right), corresponding to the case in Fig. 4(a) or decreasing in the situation of Fig. 4(b).

We also performed measurements with broader channels [b=9-10 $\mu \mathrm{m}$; cf. Fig. 1(a)], resulting in a less-pronounced minimum of the MFPT than in Fig. 2(b) and, in agreement with the above theoretical prediction, a reduced reliability to steer the particles to the right or left channel end by suitably choosing the oscillation periods $\tau$.

While the above equivalent static potential picture is useful to approximately describe the system theoretically, the actual system is different in the following important respect: All potential barriers are sampling the same sequence of configurations; only their oscillation period $\tau$ is different. Hence, there is no need to change the potential landscape in order to invert the directionality of the channel; one only has to adapt the periods $\tau$ of the oscillations.

Upon increasing the number of oscillating channel particles, the oscillation frequencies and hence the MFPT will differ less and less between adjacent channel particles. As a consequence, a continuous description in terms of a spacedependent effective diffusion coefficient will be applicable. It seems plausible that the particles will still preferably diffuse into the direction with large effective diffusion coefficient. However, the actual times to reach one of the ends of the device will obviously diverge. The quantitative details are beyond the scope of our present theoretical study, and with respect to the experiment, this limit is of minor interest.

In the case of random instead of periodic potential modulations, we still expect a resonant-activation-type minimum of the MFPT upon variation of the characteristic time scale of the potential modulations [9] similar to Fig. 2. As a consequence, the effects in Figs. 3 and 4 should qualitatively remain unchanged.

\section{CONCLUSIONS}

In this work we have demonstrated the control of effective diffusion (Sec. III B) and directed transport (Sec. IV) of colloidal Brownian particles in a channel geometry whose width is subjected to temporal oscillations with space-dependent oscillation periods. Instead of modifying the diffusion or the 
directed motion of one given particle species by properly changing the oscillation periods, as discussed in this paper, one can also modify these transport properties by using different particle species but keeping the channel oscillation parameters fixed. This opens the perspective of separating different particle species by injecting both species in the mixture into the channel and either by letting the highly diffusive species evaporate out of the channel or by collecting the two different species at the two channel ends.

We finally point out that the basic principle of our present transport mechanism is quite different from a particle pump between two reservoirs and also from directed Brownian motion due to ratchet effects $[3,14]$. In both these cases, particle transport only occurs if it passes over all potential barriers within the channel. Because within our discrete state model introduced in Sec. III B the transition rates over every given potential barrier are the same in either direction, this situation can be also described by considering equivalent static potential barriers. In this situation, however, it is obvious that no particle transport occurs, in agreement with the second law of thermodynamics. The situation considered here is somewhat different, because particles are injected into the middle of the channel and thus may proceed to one channel end without ever passing over all (in particular very high potential barriers). This eventually results in a net transport of particles in one direction.

Besides the above-mentioned potential applications for the purpose of particle sorting our present system may also be of interest in the context of active transport in biophysical systems like axons or ion pumps [1].

\section{ACKNOWLEDGMENT}

This work was financially supported by Deutsche Forschungsgemeinschaft under Be 1788 4-1, RE 1344/3-1, and SFB 613 .
[1] B. Hille, Ion Channels of Excitable Membranes (Sinauer, Sunderland, 2001); M. M. Millonas and D. R. Chialvo, Phys. Rev. Lett. 76, 550 (1996); A. Fulinski, ibid. 79, 4926 (1997); R. D. Astumian and I. Derényi, Eur. Biophys. J. 27, 474 (1998); T. Chou and D. Lohse, Phys. Rev. Lett. 82, 3552 (1999); M. J. Müller, S. Klumpp, and R. Lipowsky, J. Phys.: Condens. Matter 17, S3839 (2005); A. M. Berezhkovskii, G. Hummer, and S. M. Bezrukov, Phys. Rev. Lett. 97, 020601 (2006).

[2] Q. H. Wei, C. Bechinger, and P. Leiderer, Science 287, 625 (2000); B. Lin, B. Cui, J.-H. Lee, and J. Yu, Europhys. Lett. 57, 724 (2002); B. Cui, H. Diamant, and B. Lin, Phys. Rev. Lett. 89, 188302 (2003); M. Kollmann, ibid. 90, 180602 (2003); C. Lutz, M. Kollmann, and C. Bechinger, ibid. 93, 026001 (2004).

[3] R. Zwanzig, J. Phys. Chem. 96, 3926 (1992); G. W. Slater, H. L. Guo, and G. I. Nixon, Phys. Rev. Lett. 78, 1170 (1997); J. Han, S. W. Turner, and H. G. Craighead, ibid., 83, 1688 (1999); 86, 1394(E) (2001); C. Kettner, P. Reimann, P. Hänggi, and F. Müller, Phys. Rev. E 61, 312 (2000); D. Ruguera and M. M. Rubi, ibid. 64, 061106 (2001); Z. Siwy and A. Fulinski, Phys. Rev. Lett. 89, 198103 (2002); C. Marquet, A. Buguin, L. Talini, and P. Silberzan, ibid. 88, 168301 (2002); S. Matthias and F. Müller, Nature (London) 424, 53 (2003); R. Eichhorn and P. Reimann, Europhys. Lett. 69, 517 (2005); R. Eichhorn, P. Reimann, B. Cleuren, and C. Van den Broeck, Chaos 15, 026113 (2005); M. Streek, F. Schmid, T. T. Duong, D. Anselmetti, and A. Ros, Phys. Rev. E 71, 011905 (2005); D. Reguera, G. Schmid, P. S. Burada, J. M. Rubi, P. Reimann, and P. Hänggi, Phys. Rev. Lett. 96, 130603 (2006).

[4] E. J. W. Vervey and J. T. G. Overbeek, Theory of the Stability of Lyophobic Colloids (Elsevier, Amsterdam, 1948).

[5] Due to the hydrodynamic interaction between particle and bottom plate, the experimentally determined, one-dimensional free diffusion coefficient $D \simeq 0.24 \mu \mathrm{m}^{2} / \mathrm{s}$ is somewhat smaller than the Stokes-Einstein value $D=k_{B} T / 6 \pi \nu r \simeq 0.27 \mu \mathrm{m}^{2} / \mathrm{s}$, where $k_{B}$ is Boltzmann's constant, $T \simeq 292 \mathrm{~K}$ (room temperature), and $\nu \simeq 0.0010 \mathrm{~N} \mathrm{~s} / \mathrm{m}^{2}$ is the viscosity of water (Ref. [15]).
[6] Q. H. Wei, C. Bechinger, and P. Leiderer, Science 287, 625 (2000); B. Lin, B. Cui, J.-H. Lee, and J. Yu, Europhys. Lett. 57, 724 (2002).

[7] C. Lutz, M. Kollmann, and C. Bechinger, Phys. Rev. Lett. 93, 026001 (2004).

[8] D. Babic and C. Bechinger, Phys. Rev. Lett. 94, 148303 (2005); D. Babic, C. Schmitt, I. Poberaj, and C. Bechinger, Europhys. Lett. 67, 158 (2004).

[9] C. R. Doering and J. C. Gadoua, Phys. Rev. Lett. 69, 2318 (1992); P. Reimann and P. Hänggi, Lect. Notes Phys. 484, 127 (1997); R. N. Mantegna and B. Spagnolo, Phys. Rev. Lett. 84, 3025 (2000); J. Iwaniszewski, I. K. Kaufman, P. V. E. McClintock, and A. J. McKane, Phys. Rev. E 61, 1170 (2000); Y. Yu and S. Han, Phys. Rev. Lett. 91, 127003 (2003); C. Schmitt, B. Dybiec, P. Hänggi, and C. Bechinger, Europhys. Lett. 74, 937 (2006).

[10] Strictly speaking there are corrections quadratic in the oscillation amplitude due to the fact that the potential is a nonlinear function of the channel width $b$; see Fig. 1(a). In Sec. III B we will give an argument that these corrections are small.

[11] P. Reimann, Phys. Rev. Lett. 74, 4576 (1995); Phys. Rev. E 52, 1579 (1995).

[12] S. Lifson and J. L. Jackson, J. Chem. Phys. 36, 2410 (1962); P. Reimann, C. Van den Broeck, H. Linke, P. Hänggi, J. M. Rubi, and A. Perez-Madrid, Phys. Rev. E 65, 031104 (2002).

[13] H. Gang, A. Daffertshofer, and H. Haken, Phys. Rev. Lett. 76, 4874 (1996); M. Schreier, P. Reimann, P. Hänggi, and E. Pollak, Europhys. Lett. 44, 416 (1998); D. Reguera, P. Reimann, P. Hänggi, and J. M. Rubi, ibid. 57, 644 (2002); A. A. Dubkov and B. Spagnolo, Phys. Rev. E 72, 041104 (2005).

[14] P. Reimann, Phys. Rep. 361, 57 (2002); H. Linke (special issue guest editor), Appl. Phys. A: Mater. Sci. Process. 75, 167 (2002); J. Klafter and M. Urbakh (special issue guest editors), J. Phys.: Condens. Matter 17, S3661 (2005).

[15] M. Evstigneev, P. Reimann, C. Schmitt, and C. Bechinger, J. Phys.: Condens. Matter 17, S3795 (2005). 